\title{
Optimization of $\operatorname{Pr}_{0.9} \mathrm{Ca}_{0.1} \mathrm{MnO}_{3}$ thin films with varying in-situ oxygen annealing treatments
}

\author{
T. Elovaara ${ }^{1,2, a}$, H. Huhtinen ${ }^{1}$, S. Majumdar ${ }^{1}$, and P. Paturi ${ }^{1}$ \\ 1 Wihuri Physical Laboratory, Department of Physics and Astronomy, University of Turku, FI-20014 Turku, Finland \\ 2 The National Graduate School in Material Physics (NGSMP), Helsinki, Finland
}

\begin{abstract}
The influence of in situ oxygen annealings on narrow electronic bandwidth $\operatorname{Pr}_{0.9} \mathrm{Ca}_{0.1} \mathrm{MnO}_{3}$ films are investigated in the complex phase separation region. Measurements by x-ray diffractometry and SQUID magnetometry reveal that relatively high deposition temperature at $700^{\circ} \mathrm{C}$ relaxes the lattice by twin boundaries while the lower deposition temperature at $500^{\circ} \mathrm{C}$ with higher post-annealing temperature of $700^{\circ} \mathrm{C}$ relaxes the substrate induced strain via oxygen absorption and makes the film structure more homogeneous. This behaviour is clearly supported by the decrease of ferromagnetic ordering due to decrease of $\mathrm{Mn}^{3+}$ ions in films oxygen annealed at high temperatures and this phenomenon is widely discussed with the models of double-exchange interaction, trapping of carriers in the oxygen vacancies and formation of magnetic polarons. The results show unambiguously that because the oxygen content tailors many physical properties dramatically, the annealing treatments are in very important role when optimizing these materials for future applications.
\end{abstract}

\section{Introduction}

Perovskite manganites with general formula $\mathrm{R}_{1-x} \mathrm{~A}_{x} \mathrm{MnO}_{3}$, where $\mathrm{R}$ is a rare-earth trivalent element and $\mathrm{A}$ is a divalent alkaline-earth element, have been a subject of active research because of their complex electronic and magnetic properties, which are both physically and technologically interesting [1-5]. These correlated electron materials have many intriguing properties, such as colossal magnetoresistance (CMR), rich magnetic phase diagram including charge-ordered (CO) phase [6], first order irreversible metamagnetic transition [7] and large magnetocaloric effect [8]. These different properties also give rise to a great technological potential in applications like magnetic field sensors, memory devices $[6,10]$, magneto-optical devices, magnetic refrigeration and spintronic components [11]. This wide variety of exotic phenomena rises from strong competition of multi-electronic phases, creating coexistent phase separated regimes [4] and interplay with different phases in these materials. In these competing phase regimes the interaction between manganese ions functions differently. The double-exchange (DE) interaction between $\mathrm{Mn}^{3+}$ and $\mathrm{Mn}^{4+}$-ions via the intervening ligand oxygen ion [12] leads to ferromagnetic (FM) metallic or insulating phase, whereas the superexchange [13] interaction between $\mathrm{Mn}^{3+}$ $\mathrm{Mn}^{3+}$ and $\mathrm{Mn}^{4+}-\mathrm{Mn}^{4+}$ leads to antiferromagnetic (AFM) insulating phase. The prevailing magnetic interaction depends on the ratio between $\mathrm{Mn}^{3+}$ and $\mathrm{Mn}^{4+}$-ions and also the $\mathrm{Mn}-\mathrm{O}$ bond length and $\mathrm{Mn}-\mathrm{O}-\mathrm{Mn}$ bond angle. These properties can be affected with the use of external stimuli, like magnetic field, pressure or light, which gives these materials functionality that is very useful for the technological applications. It is possible to control the physical properties of these materials to get the wanted property more prominent by different choices of R-site trivalent and

a e-mail: tomi.elovaara@utu.fi
A-site divalent elements and $x$ values. Also the role of the oxygen in the perovskite unit cell and the oxygen vacancy related defects are very important parameters as they can be used to tailor the physical properties of the material to meet the requirements of technological applications and enable even newer applications [2].

The perovskite manganite compound $\operatorname{Pr}_{1-x} \mathrm{Ca}_{x} \mathrm{MnO}_{3}$ (PCMO) has narrow electric bandwidth $W$ [4], which distinguish it from more studied manganite compounds with broader $W$. Due the narrow $W$, PCMO is insulating in the whole doping range and it has also stable charge-ordered (CO) phase in the hole doping range of $x=0.3-0.5$. Low hole doped $\mathrm{Pr}_{0.9} \mathrm{Ca}_{0.1} \mathrm{MnO}_{3}$ is still quite unexplored, however our recent investigation showed that complex phase separated region exists in this system leading to interesting photo-induced magnetization phenomenon [1]. To use this material in the potential applications, we have systematically investigated the effect of oxygen non-stoichiometry in $\operatorname{Pr}_{0.9} \mathrm{Ca}_{0.1} \mathrm{MnO}_{3}$ thin films.

\section{Experimental details}

The films with thickness of $100 \mathrm{~nm}$ were grown on (001) $\mathrm{SrTiO}_{3}$ (STO) substrates by pulset laser deposition (PLD) technique from high purity PCMO target prepared by the solid state method [7]. The details of the deposition parameters are reported elsewhere [9]. In order to control the oxygen content of the films and affect the valency of Mn ions, different in situ growing and annealing treatments were made for four different samples. The samples A, B and $\mathrm{C}$ were deposited at $500{ }^{\circ} \mathrm{C}$ and sample $\mathrm{D}$ at $700{ }^{\circ} \mathrm{C}$. After the deposition the atmospheric pressure of $\mathrm{O}_{2}$ was introduced at $500{ }^{\circ} \mathrm{C}$ for samples $\mathrm{A}$ and $\mathrm{B}$, and at $700{ }^{\circ} \mathrm{C}$ for samples $\mathrm{C}$ and $\mathrm{D}$. After $10 \mathrm{~min}$ of annealing the samples were cooled down to room temperature with the rate $25^{\circ} \mathrm{C} / \mathrm{min}$. All the treatments are shown in the table 1 .

This is an Open Access article distributed under the terms of the Creative Commons Attribution License 2.0, which permits unrestricted use, distribution, and reproduction in any medium, provided the original work is properly cited. 
Table 1. Parameters used in in situ annealing treatments of PCMO films. In all the samples the annealing time is $10 \mathrm{~min}$ and the pressure of the $\mathrm{O}_{2}$ is $1 \mathrm{~atm}$.

\begin{tabular}{llll}
\hline Sample & Deposition $T$ & Introducing $T$ & Annealing $T\left({ }^{\circ} \mathrm{C}\right)$ \\
\hline A & 500 & 500 & 500 \\
B & 500 & 500 & 700 \\
C & 500 & 700 & 700 \\
D & 700 & 700 & 700 \\
\hline
\end{tabular}

The detailed structural measurements for the in situ treated samples were made by XRD using Philips X'pert Pro diffractometer with Schulz texture goniometer using $\mathrm{Cu} \mathrm{K} \alpha$ radiation, an incident Ni-filter, 0.04 rad Soller slits, and an $0.18^{\circ}$ thin film collimator. The crystal direction of PCMO are defined such that $a$ and $c$ forms in-plane of PCMO unit cell while the longest axis $b$ is normal to $a$ and $c$. To check the phase purity of the films, $\theta-2 \theta$ scans in $(0 b 0)$ direction were made. The lattice parameters were determined from detailed 2D $(\phi, 2 \theta)$ scan of (211) peak and $\theta-2 \theta$ scan of the peak (013) using 2D LevenbergMarquart fitting of Gaussian peaks. The peaks were chosen to avoid the overlapping of the diffraction peaks of the sample and the substrate. The out-of-plane crystallographic texture is determined by means of XRD rocking curves (RC) of PCMO (060) peaks ( $\omega$ scans).

The temperature dependence of the zero-field-cooled (ZFC) and field-cooled (FC) magnetization were measured between temperatures $5 \mathrm{~K}$ and $300 \mathrm{~K}$ with a SQUID magnetometer in $20 \mathrm{mT}, 50 \mathrm{mT}$ and $100 \mathrm{mT}$ external magnetic fields $(B)$. The magnetic hysteresis curves up to $\pm 300 \mathrm{mT}$ were measured in different temperatures between $5 \mathrm{~K}$ and $70 \mathrm{~K}$. The external field $B$ was always oriented along the plane of the films.

\section{Results}

\subsection{Structural analysis}

The measured $\theta-2 \theta$ scans for all the films showed only $(0 b 0)$ peaks of the PCMO in addition to the substrate peaks. Therefore no impurities were detected. The lattice parameters were determined from the difference of the $(0 b 0)$ peaks and $2 \theta$ scans of the (211) and (013) peaks. The values are shown in table 2. For the samples A, B, and $\mathrm{C}$ there is no change in unit cell volume within error bars, but sample D has clearly smaller unit cell size according to the peak positions. This decrease in the unit cell volume seems to be due the oxygen content of the film. The shortening of the unit cell parameters due oxygen absorption is well explained by the content of $\mathrm{Mn}^{4+}$ ions compared to that of $\mathrm{Mn}^{3+}$, since the cation-cation bond is longer than cationoxygen-cation bond [13]. As the ionic radius of $\mathrm{Mn}^{3+}(0.07$ $\mathrm{nm})$ exceeds that of $\mathrm{Mn}^{4+}(0.05 \mathrm{~nm})$, each replacement of $\mathrm{Mn}^{3+}$ by $\mathrm{Mn}^{4+}$ reduces the volume of the unit cell. To maintain the balance of the charge, each oxygen ion oxidizes two $\mathrm{Mn}^{3+}$ ions to $\mathrm{Mn}^{4+}$ causing a decrease of 0.04 $\mathrm{nm}$ in the lattice parameters. Opposite to this, when oxygen is added in the $\mathrm{MnO}_{6}$ octahedron, there is an increase of $1 / 6$ of oxygen ionic radius $(0.12 \mathrm{~nm})$ i.e. $0.02 \mathrm{~nm}$ in the lattice parameter. In the former process, which is more prominent, the lattice parameters decrease with increasing oxygen content. The increase in the $\mathrm{Mn}^{4+}$ content also reduce the cooperative Jahn-Teller (JT) [14] distortion affecting the $\mathrm{Mn}^{3+}$ ions, generating local JT distortion around the $\mathrm{Mn}^{3+}$ ions [15]. This increases the ordering of PCMO crystal at the same time packing the atoms into a smaller volume [7].

The in-plane lattice parameters of the STO substrates are greater than those of bulk PCMO $\left(d_{101}=3.897 \AA\right.$ [7] for the bulk, to be compared to $3.905 \AA$ for the STO substrate) and thus the deposited films are under tensile strain. In spite of the tensile strain, the in-plane lattice parameters of the films are smaller than the bulk values $(a=5.451 \AA$, $b=7.662 \AA$ and $c=5.572 \AA$ [7]) indicating that the effect of oxygen is greater than the effect of tensile strain. It is also good to notice that due to the tensile strain by the substrate, the difference of the unit cell volume compared to bulk, $\Delta V$, of PCMO is reduced. The amount of the strain in the lattice and the variation of the lattice parameters due to lattice defects are seen from the width of the peak full width at half-maximum (FWHM). The FWHM values for (060) peaks are shown in table 2; for the other peaks, the changes were in the same direction and of the same size. For the sample A, the $2 \theta$ FWHM value is the highest and the difference of unit cell volume compared to bulk value is smallest. This indicates that the tensile strain and the amount of the defects in the lattice are the highest of all the samples. This is due to the low deposition temperature where the lattice does not relax as at higher deposition temperatures. Also in the low temperature in situ annealing treatment, the amount of absorbed oxygen is smaller than at higher temperatures inducing more oxygen vacancies to the sample. The samples B and C have grown fairly relaxed having small FWHM values due to high temperature annealing treatment. The high FWHM value of the sample D compared to samples B and C can be explained with higher oxygen gradient between bulk and surface. The changes due to conversion of $\mathrm{Mn}^{3+}$ to $\mathrm{Mn}^{4+}$ are likely to be more pronounced at the surface of the film than near the substrate interface, which again increases the distribution of the volume in PCMO unit cells.

The $2 \theta-\phi$ scans of the (211) peaks are shown in figure 1 . It is clearly seen that sample D has twin boundaries. The reason for the existence of twin boundaries is probably due to the high deposition temperature which improves the oxygen absorption and also partly relaxes the lattice during deposition process. In this case the reduction of lattice size is more prominent and lattice mismatch between film and substrate increases. The peak widths in the $\phi$ direction are also listed in table 2 . As for $2 \theta$, the highest $\phi$ FWHM value is for the sample A indicating highest variations in the inplane lattice direction. It is worth noticing that for the main peak of the sample D the FWHM value is very small indicating very relaxed sample. The lattice coherence length $r_{c}$ is calculated from $r_{c}=b / \pi k \Delta \omega$, where $b$ is the lattice parameter, $k$ is the order of the Bragg reflection and $\Delta \omega$ is the FWHM of the rocking curve [16]. It can be seen that for the D sample the $r_{c}$ value is the highest, indicating the long range ordering in the $b$ direction. Also the $\mathrm{B}$ sample has higher $r_{c}$ value than that of $\mathrm{A}$ and $\mathrm{C}$ samples. The obtained $r_{c}$ values are typical for pulsed laser deposition films [17], indicating that the growth process of the films is normal. 
Joint European Magnetic Symposia 2012

Table 2. Structural parameters of the films determined from the XRD measurements. $\Delta V$ is the difference of unit cell volume compared to bulk value of $232.73 \AA^{3}$ [7]. The number in parentheses is the error of the last digit.

\begin{tabular}{llllll}
\hline Film & $a(\AA)$ & $b(\AA)$ & $c(\AA)$ & $V\left(\AA^{3}\right)$ & $\Delta V(\%)$ \\
\hline A & $5.446(8)$ & $7.660(6)$ & $5.532(9)$ & $230.8(9)$ & $-0.8(4)$ \\
B & $5.436(8)$ & $7.662(6)$ & $5.546(9)$ & $231.0(9)$ & $-0.7(4)$ \\
C & $5.437(8)$ & $7.667(6)$ & $5.516(9)$ & $230.0(9)$ & $-1.2(4)$ \\
D & $5.416(8)$ & $7.659(6)$ & $5.495(9)$ & $227.9(9)$ & $-2.1(4)$ \\
\hline Film & $2 \theta$ FWHM $(060)\left({ }^{\circ}\right)$ & $\phi$ FWHM $(211)\left({ }^{\circ}\right)$ & $\omega$ FWHM $(060)\left({ }^{\circ}\right)$ & $r_{c}(\mathrm{~nm})$ & \\
\hline A & $0.68(2)$ & $1.30(3)$ & $0.297(3)$ & $7.84(8)$ & \\
B & $0.50(1)$ & $0.89(2)$ & $0.281(2)$ & $8.30(6)$ & \\
C & $0.56(1)$ & $0.98(2)$ & $0.307(3)$ & $7.60(8)$ & \\
D & $0.60(1)$ & $0.70(6)$ & $0.208(1)$ & $11.22(6)$ & \\
\hline
\end{tabular}

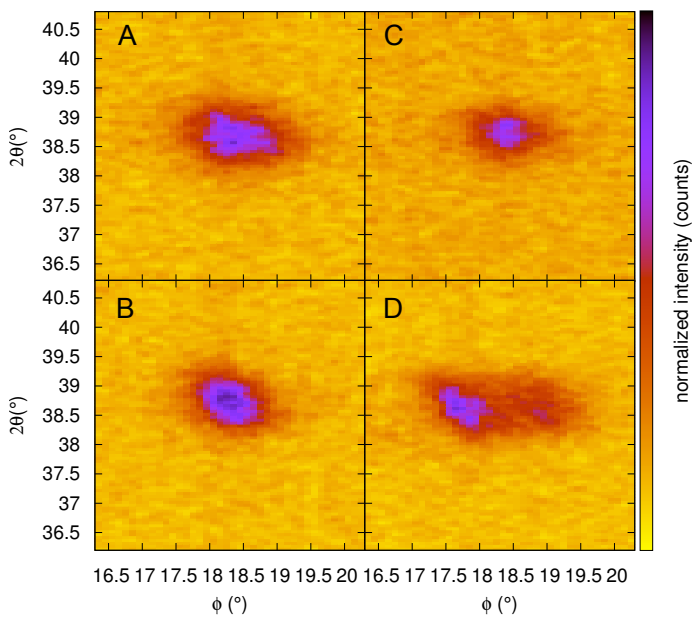

Fig. 1. (211) peaks as a function of $2 \theta$ and $\phi$ for the in situ treated films A, B, C and D.

Even though the above presented structural changes as oxygen deficiency, substrate induced strain, crystal defects and twins are very difficult to distinguish from each other, the overall effect of the in situ annealing treatments in the lattice structure can be explained as follows. In the low temperature deposition and annealing (sample A) the film has grown uneven due to oxygen vacancy related defects and high tensile strain in the in-plane direction. When the temperature is increased to $700{ }^{\circ} \mathrm{C}$ in post-annealing treatment (sample B and C) more oxygen is adsorbed into the film and the lattice is relaxed and more uniform. In the case where the sample is deposited and annealing treated at 700 ${ }^{\circ} \mathrm{C}$ (sample D), the lattice is partly relaxed during the deposition process causing twin boundaries and eventually very relaxed lattice parameters.

\subsection{Magnetic properties}

To observe the effect of annealing treatments on the magnetic phases, the $M_{Z F C}(T)$ and $M_{F C}(T)$ curves were measured in different external magnetic fields for all the samples A, B, C and D. The measurement in $50 \mathrm{mT}$ magnetic field can be seen in figure 2. A significant difference between ZFC and FC magnetizations is observed for all the in situ annealing treatments indicating the background AFM ordered ground state with a frustrated FM clusters in it, which leads to absence of the long-range FM ordering and presence of a spin/cluster glass phase [18].
$M(T)$ magnetization gives information of the characteristic features like Curie-temperature $T_{C}$, a maximum in the $M_{Z F C}(T)$ curves around a freezing temperature $T_{f}$ and an irreversibility temperature $T_{g}$, where $\mathrm{ZFC}$ and FC branches start to deviate. As can be seen from the figure 2, the magnetization in the sample A is significantly higher than in other three samples. The second highest magnetization of all the samples is in the sample D. Also for the samples $\mathrm{A}$ and $\mathrm{D}$ the onset $T_{C}$ is higher, around $120 \mathrm{~K}$, and the PM-FM transition is much sharper than in the other two samples. For the sample D the shape of the $M(T)$ curves is different than that for the samples A, B and C, where the $M_{Z F C}(T)$ starts to grow steeply at the start, as the FM clusters grow larger, settling up a broad maximum. As the $M_{Z F C}(T)$ curve for the sample D grows slowly at the start and then makes a narrow peak. Actually in the curve measured at $100 \mathrm{mT}$ field for sample D, there appears a second maxima around $15 \mathrm{~K}$ (not shown here). Appearance of two maxima in the $M_{Z F C}(T)$ curve refers to existence of two magnetic phases in the ground state. Also a dell in the $M_{F C}(T)$ curve indicates magnetic non-homogeneity, which is seen in all the measured fields. This magnetic impurity phase probably causes the higher $T_{C}$ and magnetization values for sample D. It seems that observed twin boundaries in structure analysis also cause a second magnetic phase in the magnetic ground state. With increasing magnetic field, the peak in the $M_{Z F C}(T)$ curves broadens and shifts to lower temperature and irreversibility magnetization starts to decrease in all the samples, signifying improved FM ordering. These effects are most prominent for the sample A indicating the best FM ordering among the four samples.

The rather large change in the magnetic moment of the sample A compared to the other samples can be explained with the oxygen content of PCMO. The net magnetic moment decreases as the oxygen content increases in the sample decreasing the concentration of the $\mathrm{Mn}^{3+}$ ions with higher magnetic moment. Also in the sample A, the substrate induced tensile strain is highest. The tensile strain can unbend the $\mathrm{MnO}_{6}$ octahedron and straighten the $\mathrm{Mn}-\mathrm{O}-\mathrm{Mn}$ bond angles which broadens the electron bandwidth $W[6]$ and enables the charge carriers to move easily between adjacent $\mathrm{Mn}^{3+} / \mathrm{Mn}^{4+}$ sites, reducing orbital localization and improving the FM interaction [19]. The oxygen vacancies can also improve the FM orientation of the film, as the unpaired electrons can be trapped to them and form self-trapped rigid magnetic polarons, which can result a local FM ordering [20]. The increase in the oxygen content of the samples B, C and D decreases the JT distortion 


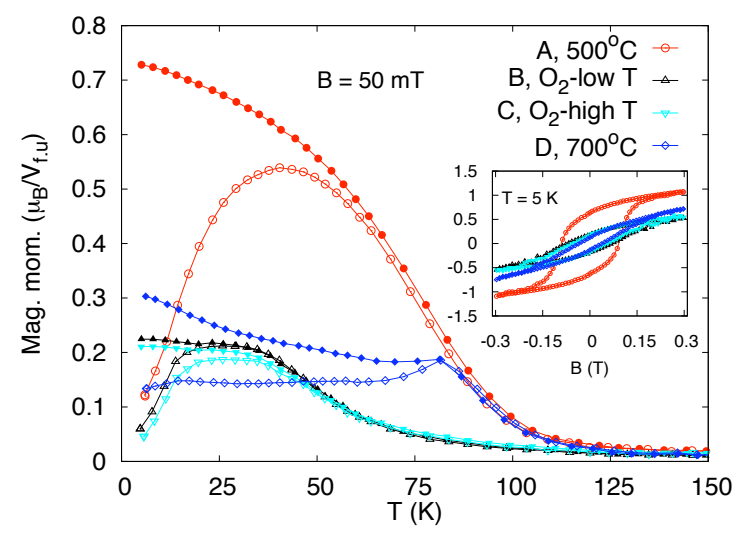

Fig. 2. The temperature dependence of the $M_{Z F C}$ (open symbols) and $M_{F C}$ (solid symbols) magnetization for all the samples measured at $50 \mathrm{mT}$ magnetic field. The inset shows magnetic hysteresis loops $M(B)$ between -300 and $300 \mathrm{mT}$ field at $5 \mathrm{~K}$.

which also unbends the $\mathrm{MnO}_{6}$ octahedron and straightens the Mn-O-Mn angle. This can also increase FM ordering, but clearly the loss of $\mathrm{Mn}^{3+}$ ions, tensile strain and oxygen vacancies have more effect on net magnetic moment and FM ordering.

The inset of figure 2 shows the magnetic hysteresis loops $M(B)$ measured at $5 \mathrm{~K}$ for all the samples. The saturation magnetization $M_{\text {sat }}$ is highest for the sample A indicating higher FM volume fraction. For none of the samples is the magnetization fully saturated in $0.3 \mathrm{~T}$ field, which also indicates the AFM background. Samples B, C, and $\mathrm{D}$ show narrow loops, when the opening in the sample A is much broader and makes a sharper magnetic transition. This is probably due to the strain between AFM-FM coupling interfaces. As in the sample A, the FM volume fraction is higher compared to other samples, and the strains at the interfaces between the AFM and FM regions are comparatively weak, leading to sharper magnetic transition. The coercive fields $B_{c}$ for the samples A, B, C and $\mathrm{D}$ are $90 \mathrm{mT}, 70 \mathrm{mT}, 55 \mathrm{mT}$ and $36 \mathrm{mT}$ respectively. This difference in the coercive field values can be due to oxygen vacancy related defects, which pin the domain wall movements i.e. more oxygen vacancies in the sample the higher the coercive field.

\section{Conclusions}

The structural and magnetic properties of four differently in situ oxygen annealing treated $\operatorname{Pr}_{0.9} \mathrm{Ca}_{0.1} \mathrm{MnO}_{3}$ samples have been studied. The sample A with lowest deposition and oxygen annealing temperature at $500{ }^{\circ} \mathrm{C}$ has the lowest oxygen content of all the samples, with most oxygen related defects and highest tensile lattice strain. It is clear that A sample has the highest overall magnetic moment and the shape of the $M(T)$ curves are very similar as the bulk material of $\operatorname{Pr}_{0.9} \mathrm{Ca}_{0.1} \mathrm{MnO}_{3}$ [7], which makes it the most optimal of all the four films. As the oxygen content in the sample increases, with higher temperature $\left(700{ }^{\circ} \mathrm{C}\right)$ oxygen annealing treatment, the lattice becomes more relaxed and the FM ordering in the sample is reduced. The difference in the magnetic properties between sample B and $\mathrm{C}$ is minute, indicating that the effect of the oxygen introducing temperature in the oxygen annealing treatment is very small to magnetic properties. The reason for decreasing FM ordering in the sample as the oxygen content increases is mainly due the decrease of the $\mathrm{Mn}^{3+}$ ions and the oxygen vacancies. The stronger tensile strain can also unbend the Mn-O-Mn bond angles strengthening the doubleexchange interaction between $\mathrm{Mn}^{3+}$ and $\mathrm{Mn}^{4+}$ ions, leading to higher ferromagnetism. Also the trapping of electrons at the oxygen vacancies leading to the formation of rigid magnetic polarons can increase magnetic moment, with decreasing oxygen content. When the film (sample D) is prepared with high deposition temperature at 700 ${ }^{\circ} \mathrm{C}$, the lattice is partly relaxed during the deposition process inducing reduced unit cell size and twin boundaries to the lattice. This lattice twinning is also seen in magnetic measurements as a magnetic second phase impurity, which probably causes the higher magnetic moment and $T_{C}$ values in sample D than in samples B and C.

\section{References}

1. S. Majumdar, H. Huhtinen, M. Svedberg, P. Paturi, S. Granroth and K. Kooser, J. Phys. Cond. Mat. 23, 466002 (2011).

2. B. Bryant, C. Renner, Y. Tokunaga, Y. Tokura and G. Aeppli, Nat. Comm. 2, 212 (2011).

3. A. Asamitsu, Y. Tomioka, H. Kuwahara and Y. Tokura, Nature 388, 50 (1997).

4. A. Dagotto, T. Hotta and A. Moreo, Physics Reports 344, 1 (2001).

5. A.-M. Haghiri-Gosnet and J.-P. Renard, J. Phys. D.:Appl. Phys. 36, R127 (2003).

6. Y. Tokura, Colossal magnetoresistive oxides (Gordon and Breach Science, New York, 2000).

7. T. Elovaara, H. Huhtinen, S. Majumdar and P. Paturi, J. Phys. Cond. Mat. 24, 216002 (2012).

8. V. S. Kolat, T. Izgi, A. O. Kaya, N. Bayri, H. Gencer and S. Atalay, J. Magn. and Magn. Mater. 322, 427 (2010).

9. M. Svedberg, S. Majumdar, H. Huhtinen, P. Paturi and S. Granroth, J. Phys. Cond. Mat. 23, 386005 (2011).

10. Y. Murakami, H. Kasai, J. J. Kim, S. Mamishin, D. Shindo, S. Mori and A. Tonomura, Nature Nanotechnology 5, 37 (2010).

11. M. Bibes and A. Barthelemy, IEEE T. Electr. Dev. 54, 1003 (2007).

12. C. Zener, Physical Review 81, 440 (1951).

13. J. B. Goodenough, Physical Review 100, 564 (1955).

14. H. A. Jahn and E. Teller, Proc. R. Soc. A 161, 220 (1937).

15. F. Duan and J. Guojun, Introduction to Condensed Matter Physics (World Scientific Publishing, 2005).

16. A. Gauzzi and D. Pavuna, Appl. Phys. Lett. 66, 1836 (1995).

17. H. Huhtinen, K. Schlesier and P. Paturi, Supercond. Sci. Technol. 22, 075019 (2009).

18. S. L. Young, H. Z. Chen, L. Horng, J. B. Shi and Y. C. Chen, Jpn. J. Appl. Phys. 40, 4878 (2001).

19. W. Prellier, A. M. Haghiri-Gosnet, B. Mercey, P. Lecoeur, M. Hervieu, C. Simon and B. Raveau, Appl. Phys. Lett. 77, 1023 (2000).

20. T. Suominen, H. Huhtinen, S. Majumdar, P. Paturi, V. S. Zakhvalinskii and R. Laiho, J. Phys. Cond. Mat. 21, 266001 (2009). 